\title{
Judging statistical significance by inspection of standard error bars
}

\author{
WILLIAM P. DUNLAP \\ Tulane University, New Orleans, Louisiana \\ and \\ JAMES G. MAY \\ University of New Orleans, New Orleans, Louisiana
}

\begin{abstract}
This paper describes the evaluation of statistical significance by visual comparison of the distance between means relative to the lengths of standard error bars in graphic representations of data. The relationship between the $t$ statistic and means separated by various numbers of standard error bars permits the observer to make quick conservative judgments of the significance of differences between means without computations or extensive tables. When standard error bars touch or overlap, the means do not differ significantly. Means that differ by three times the length of a standard error bar differ significantly at the .05 level as long as the sample size per mean is nine or more.
\end{abstract}

Recently, the question was broached by a reviewer as to whether the appropriate designator for dispersion in a graphical depiction of experimental results was standard deviation $(s)$ bars or standard error $(S E)$ bars, where $S E=$ $s / n^{1 / 2}$. The reviewer pointed out that Glantz (1981) settled this argument in favor of the former with the pristine logic that only $s$ provides information from which one might generalize to the population. Notwithstanding Glantz's contention (and perhaps partly because our figures were already drawn and photographed using $S E$ bars), we argued for the use of $S E$ bars because they permit the reader to graphically infer significance. The substance of this short note is concerned with how statistical significance can be judged by inspection of graphs with $S E$ bars.

\section{Rationale}

Student's $t$ test for the difference of two independent means can be expressed as

$$
t=\left(\bar{x}_{1}-\bar{x}_{2}\right) /\left(2 s^{2} / n\right)^{1 / 2},
$$

where $s^{2}$ is the estimated common variance and $n$ is the sample size per mean. The standard error of a mean can be written as

$$
S E=\left(s^{2} / n\right)^{1 / 2} ;
$$

therefore, the $t$ statistic can be rewritten as

$$
t=\left(\bar{x}_{1}-\bar{x}_{2}\right) /\left(2^{1 / 2} S E\right)=0.707\left(\bar{x}_{1}-\bar{x}_{2}\right) / S E .
$$

The latter part of this expression, the number of times

Address correspondence to William P. Dunlap, Department of Psychology, Tulane University, 2007 Percival Stern Hall, New Orleans, LA 70118.
$S E$ goes into the interval $\left(\bar{x}_{1}-\bar{x}_{2}\right)$, thus represents the number of $S E$ bars separating two means on a graph. The degrees of freedom for $t$ are $2(n-1)$.

Table 1 shows the $t$ statistic resulting from means separated by various numbers of $S E$ bars. To the right in Table 1 are presented minimal sample sizes per mean for which the $t$ value is significant at various significance levels. Normally, $S E$ bars are depicted bidirectionally about the mean, but we have graphed them to accentuate the relative comparison between the means.

Table 1 can be interpreted as follows: (1) If the $S E$ bars for two means touch or overlap, those means do not differ significantly, because the number of $S E$ bars separating them is two or less (see A in Figure 1); (2) means separated by three $S E$ bars (see B in Figure 1) are significant at the .05 level if the sample size per mean is 9 or more; (3) means separated by four $S E$ s are significant at the .05 level if $n$ is 3 or more (see C in Figure 1), and at the .01 level if $n$ is 12 or more (see D in Figure 1); and so forth. Alternatively, assuming there are 10 or more scores per mean, one can treat three $S E$ bars as the $5 \%$ critical difference, a bit more than four $S E$ bars as the $1 \%$ critical difference, and six $S E$ bars as the $0.1 \%$ critical difference between means (see $E$ in Figure 1).

Table 1

Values of $t$ and Minimal Sample Sizes for Significance for Means Separated by Various Numbers of Standard Error (SE) Bars

\begin{tabular}{ccccc}
\hline \multirow{2}{*}{$\begin{array}{c}\text { No. of } S E \text { Bars } \\
\text { Between Means }\end{array}$} & $t$ & \multicolumn{3}{c}{ Significance Level } \\
\cline { 3 - 5 } 2 & 1.414 & n.s. & n.s. & n.s. \\
3 & 2.121 & 9 & n.s. & n.s. \\
4 & 2.828 & 3 & 12 & n.s. \\
5 & 3.536 & 3 & 5 & 23 \\
6 & 4.243 & 3 & 4 & 8 \\
\hline
\end{tabular}




\section{MEANS AND STANDARD ERROR BARS}

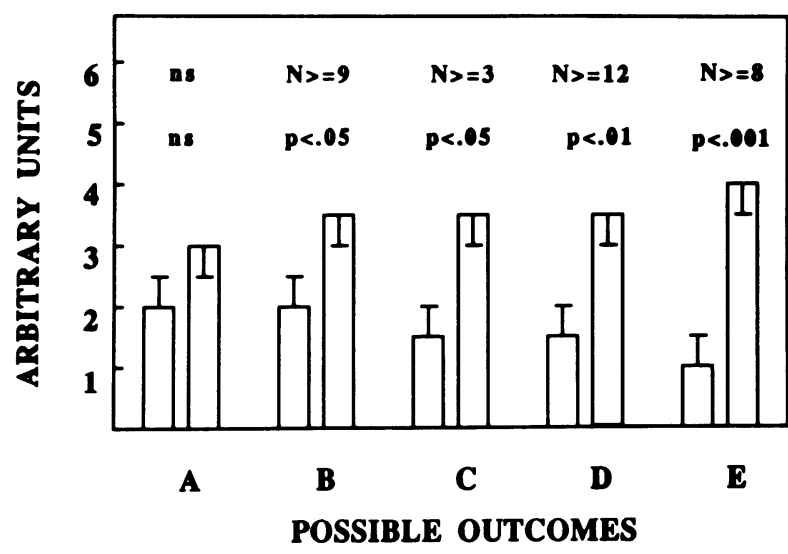

Figure 1. Examples of means separated by various numbers of standard error $(S E)$ bars, sample sizes $(N)$, and significance levels (p). Means in examples A, B, C, D, and E are separated by 2, 3, 4,4 , and $6 S E$ bars, respectively.

\section{Caveats}

$S E$ bars associated with different means are seldom the same length, so how does one proceed to judge the number of $S E$ bars separating a pair of means when they are unequal? A conservative step is to use the larger $S E$ bar as the unit of measurement. On the other hand, it is probably fairly safe to use an average $S E$ bar to make such judgments. This is particularly true when the larger $S E$ bar is associated with the larger mean, because such a condition suggests skew in the underlying data, and tests of significance tend to be conservative with skewed data (Levine \& Dunlap, 1982). What if the $n$ s differ? Again, the conservative approach would be to use the smaller $n$; however, it is probably safe to use the average $n$.

The significance judgment procedure described above was based on a $t$ test for independent means, so what if the means presented are based on repeated measures data where the data are not independent? In these cases, it is usually true that positive correlations will exist from measure to-measure, in which case the appropriate error term will be smaller than the error term for independent means. Therefore, in most cases, the use of Table 1 in assessing significance will be conservative when the data are repeated measures; power will be lost, but at least the test is safe. In the rare event of a negative correlation the visual test would be too liberal.

\section{Discussion}

$S E$ bars on plots of experimental means enable the reader to make quick judgments regarding significance. Because such judgments are based on the $t$ statistic, these significance decisions are analogous to the least significant difference test, originally proposed by Fisher (1935), who suggested following significant analysis of variance findings by individual $t$ tests. Hence, it is assumed that the experimental means depicted in a figure were plotted because analysis of variance had shown significant overall differences or interactions.

It is true that more sophisticated procedures, such as range tests (Miller, 1966), exist that permit more precise tests of significance of pairwise comparisons among means. On the other hand, depicting significance derived from a range test is often difficult to incorporate into a figure.

The use of $S E$ bars in figures, which permits quick approximate judgments of significance, should be taken in the spirit of what has been called Tukey's pocket test (Tukey, 1959). Like that test, the use of $S E$ bars is a highly compact procedure, requiring no complex arithmetic and no extensive significance tables (other than the few values presented in Table 1), which, at the sacrifice of some precision, can be applied quickly and easily to make significance judgments.

\section{REFERENCES}

FISHER, R. A. (1935). The design of experiments. London: Oliver \& Boyd.

Glantz, S. (1981). Primer of biostatistics. New York: McGraw-Hill. Levine, D. A., \& DunlaP, W. P. (1982). Power of the $F$ test with skewed data: Should one transform or not? Psychological Bulletin, 92, 272-280.

MiLlER, R. G. (1966). Simultaneous statistical inference. New York: McGraw-Hill.

TUKEY, J. W. (1959). A quick, compact, two-sample test to Duckworth's Specifications. Technometrics, 1, 31-48.

(Manuscript received June 6, 1988.) 